\title{
Young Children Who Eat Animal Sourced Foods Grow Less Stunted: Findings of Contemporaneous and Lagged Analyses from Nepal, Uganda and Bangladesh
}

Sonia Zaharia ( $\nabla$ sonia.zaharia@tufts.edu )

Friedman School of Nutrition Science and Policy, Tufts University https://orcid.org/0000-0002-10117582

Shibani Ghosh

Friedman School of Nutrition Science and Policy, Tufts University

Robin Shrestha

Friedman School of Nutrition Science and Policy, Tufts University

Swetha Manohar

Johns Hopkins Bloomberg School of Public Health

Andrew Thorne-Lyman

Johns Hopkins University https://orcid.org/0000-0001-5917-4126

Bernard Bashaasha

Makerere University

Nassul Kabunga

Feed the Future Innovation Lab for Nutrition

Sabi Gurung

Johns Hopkins Bloomberg School of Public Health

Grace Namirembe

Friedman School of Nutrition Science and Policy, Tufts University

Katherine Heneveld

Friedman School of Nutrition Science and Policy, Tufts University

Lichen Liang

Friedman School of Nutrition Science and Policy, Tufts University

Patrick Webb

Tufts University, Medford, United States https://orcid.org/0000-0002-9857-3354

Article

Keywords: animal-sourced food, child growth, anthropometric outcome 
Posted Date: September 23rd, 2020

DOl: https://doi.org/10.21203/rs.3.rs-74484/v1

License: (c) (i) This work is licensed under a Creative Commons Attribution 4.0 International License. Read Full License

Version of Record: A version of this preprint was published at Nature Food on April 21st, 2021. See the published version at https://doi.org/10.1038/s43016-021-00259-z. 
20

\section{Young Children Who Eat Animal Sourced Foods Grow Less Stunted: Findings of Contemporaneous and Lagged Analyses from Nepal, Uganda and Bangladesh}

Sonia Zaharia ${ }^{1,2, *}$, Shibani Ghosh ${ }^{1,2}$, Robin Shrestha ${ }^{1,2}$, Swetha Manohar ${ }^{2,3}$, Andrew L ThorneLyman $^{2,3}$, Bernard Bashaasha ${ }^{2,4}$, Nassul Kabunga ${ }^{2}$, Sabi Gurung ${ }^{2,3}$, Grace Namirembe ${ }^{1,2}$, Katherine Heneveld ${ }^{1,2}$, Lichen Liang ${ }^{1,2}$, and Patrick Webb ${ }^{1,2}$

1. Friedman School of Nutrition Science and Policy, Tufts University, Boston, MA, USA

2. Feed the Future Innovation Lab for Nutrition

3. Johns Hopkins Bloomberg School of Public Health, Baltimore, MD, USA

4. Makerere University, Kampala, Uganda 4

To whom correspondence should be addressed: Dr. Sonia Zaharia, Address: 150 Harrison Ave, Boston, Massachusetts; Email: Sonia.Zaharia@tufts.edu 


\section{Abstract}

22

23 In resource constrained countries, animal-sourced foods (ASFs) are an important nutrient-dense

24 source of vitamins, minerals and macronutrients. While several studies have suggested the value 25 of ASFs to child growth, most empirical evidence is based on cross-sectional data which can

26 only provide information about the contemporaneous relationship between diet and

27 anthropometric outcomes. This study uses longitudinal panel data for Nepal, Bangladesh, and

28 Uganda to assess the association between contemporaneous as well as past ASF consumption

29 and linear growth of children aged 6-24 months. Fixed effects models found that ASF

30 consumption was significantly correlated with lower stunting, with a decline in stunting

31 prevalence as high as $10 \%$ in Nepali children who had consumed any ASF in the previous year.

32 Consuming two or more ASFs showed an even higher magnitude of association, ranging from a

$3310 \%$ decline in prevalence of stunting associated with lagged consumption in Bangladesh to a

$34 \quad 16 \%$ decline in Nepal.

35

36 
37 In 2020, despite improving trends globally, there are an estimated 144 million stunted children

38 under 5 years of age $(\sim 22 \%)$. The largest burden is in South Asia and Sub-Saharan Africa, which

39 together account for over $85 \%$ of global prevalence ${ }^{1,2}$. Child stunting may adversely affect

40 physical and cognitive development, future health, income earnings and labor productivity ${ }^{3,4}$.

41 While many factors contribute to stunting, including in utero insults, poor birth outcomes,

42 inadequate caring practices, and infections, a low-quality diet, including a lack of sufficient

43 energy, vitamins, minerals (zinc, iron), quality protein and essential amino acids is important,

44 given its role in supporting optimal child growth $5,6,7,8$.

46 Several multi-country studies and supplementation trials have investigated the relationship

47 between ASFs and stunting. In a 180-country analysis, Ghosh et al. (2012) ${ }^{9}$ demonstrated that

48 the quality of protein consumed (ASF versus plant-based) was significantly associated with the

49 prevalence of stunting. Using Demographic and Health Survey data from multiple countries,

50 Krasevec et al. $(2017)^{10}$ and Headey et al. (2018) ${ }^{11}$ found that eating more than one type of any

51 ASF was associated with a reduction in stunting in young children. Trials and observational

52 studies have generated mixed evidence of the association between ASF consumption and child

53 growth in different contexts. While many studies reported a positive association between linear

54 growth in children and consumption of dairy ${ }^{12,13,14}$, meat ${ }^{15,16,17}$, fish ${ }^{18,19}$, and eggs ${ }^{20,21}$, some

55 did not find significant effects ${ }^{22}, 23$.

57 Despite documented associations, most multi-country studies are cross-sectional and test the 58 contemporaneous relationship between diet and stunting. This analysis responds to an important 59 gap. It tests empirically the hypothesis that ASF consumption is associated with reduced stunting 
over time. Using large longitudinal panels from three low-income countries (Nepal, Bangladesh

61 and Uganda), we examined anthropometric outcomes (stunting and length-for-age z-scores) in

62 relation to both contemporaneous and past consumption of various ASFs by young children,

63 controlling for relevant confounding factors. We made use of repeated observations on the same

64 child to test both contemporaneous and lagged effects.

65

66 We found a significant positive association between a child's ASF consumption and length-for-

67 age z-score (LAZ), and a negative association with stunting. This holds both for

68 contemporaneous and lagged ASF consumption, with the strongest results observed in relation to

69 lagged effects. This outcome makes biological sense given that child growth is a cumulative

70 process. Our findings suggest an aggregating benefit to past and ongoing ASF consumption on

71 length-for-age z-scores and reduced stunting. Confirming findings from Headey et al. $(2018)^{11}$,

72 we found the association between ASF consumption and LAZ/stunting was larger if two or more

73 different types of ASFs were consumed. A critical finding not reported previously is that while

74 dairy was positively correlated with growth across the three different countries, other ASFs were

75 also associated with growth outcomes and these differed by country, with meat in Nepal, fish in

76 Uganda, and eggs in Bangladesh.

77

$78 \quad$ Results

79 Models were estimated using fixed effects (FE) panel regressions and are presented in Tables 2-

80 4. All models adjust for consumption of other food groups, age, gender, illness, maternal height

81 and education, and household sanitation. In alternative specifications that include household

82 wealth index instead of mother's education and household sanitation, most, but not all results 
83 hold. Our preferred specification uses education and sanitation which are correlated with a 84 household's wealth index but more proximal correlates of stunting. In each table, Panel A 85 presents results on LAZ and Panel B on stunting. Any Contemporaneous ASF Consumption and LAZ or Stunting Contemporaneous consumption of any ASF was positively associated with LAZ in all three countries (Table 2), adjusting for district-survey round fixed effects, other food groups and control variables. In Nepal and Uganda, we also found a negative association with stunting in infants aged 6 to 12 months.

In Nepal, LAZ scores were significantly higher $(\beta=0.12, \mathrm{p}<0.1,95 \% \mathrm{CI}(-0.03,0.27))$ in infants and young children aged 6 to 24 months who consumed any ASF relative to those who did not, 95 adjusting for consumption of other food groups, age, gender, illness, maternal height and education, household sanitation, and time-varying district characteristics. Coefficients for other

97 food groups are presented in Supplementary Table 4. Dark green leafy vegetables, vitamin A98 rich fruits and vegetables, and legumes, nuts and seeds were all associated with better LAZ. This speaks to the important point that different foods contain different types and concentrations of key vitamins and minerals and that diet diversification matters not just because of including one or other food group, but to enhance the range and quality of foods consumed every day. Using 102 child and survey round fixed effects (Supplementary Table 4, Panel A, model 2) there was a 103 stronger association between LAZ and any ASF consumption $(\beta=0.11, \mathrm{p}<0.05,95 \% \mathrm{CI}(0.03$, 104 0.19)). Disaggregated by age group, consumption of any ASF by Nepali infants 6-12 months was 105 associated with a 0.23 higher LAZ $(\mathrm{p}<0.05,95 \%$ CI $(0.07,0.39)$, Supplementary Table 4, Panel 
A, Model 5). We found a negative association between ASF consumption and stunting in infants aged 6-12 months with a 5\% drop in prevalence associated with any ASF consumption $(\mathrm{p}<0.05$, 95\% CI (-0.09, -0.02), Supplementary Table 4, Panel B, Model 5).

In Bangladesh, infants and young children aged 6 to 24 months who consumed any ASF had significantly higher LAZ scores $(\beta=0.12, \mathrm{p}<0.01,95 \% \mathrm{CI}(0.05,0.19))$ than those who did not (Table 2, Panel A). In models disaggregated by age group, the contemporaneous association between ASF consumption and LAZ in the 13 to 24 months age group was positive and statistically significant $(\mathrm{p}<0.05)$. The Z-score of those who consumed any ASF was 0.14 SD higher than with no ASF $(95 \%$ CI $(0.03,0.25)$, Supplementary Table 5, Panel A, model 7). We also found a positive association $(\beta=0.15, \mathrm{p}<0.1,95 \% \mathrm{CI}(-0.01,0.32))$ between LAZ and staples consumption but not with other food groups. ASF consumption was relatively high in Bangladesh, some of which was in the form of fish, which might explain why the additional impact on nutrition of consuming other food groups was not significant.

In Uganda, LAZ was 0.14 Z-scores higher $(\mathrm{p}<0.1,95 \% \mathrm{CI}(-0.00,0.28))$ in children aged 6 to 24 months who consumed any ASF (Table 2, Panel A). The results appear to be driven by the 13 to 24 months age group, where the LAZ was $0.2 \mathrm{Z}$-scores higher $(\mathrm{p}<0.05,95 \% \mathrm{CI}(0.04,0.37))$ in those that consumed any ASF versus those that did not (Supplementary Table 6, Panel A, Model 5). We found a negative association between stunting and ASF consumption in the Ugandan children aged 6-24 months $(\beta=-0.03, \mathrm{p}<0.1,95 \% \mathrm{CI}(-0.07,0.01)$, Table 2$)$ which appears to be driven by the statistically significant coefficient obtained in the 6-12 months age group ( $(=-0.09$, p<0.01, 95\% CI (-0.15,-0.04), Supplementary Table 6, Panel B, Models 1 and 3). 


\section{Number of ASFs and LAZ or Stunting}

Consumption of two or more different types of ASFs had a stronger association with LAZ and stunting than just one type in all countries. In Nepal, infants and young children that consumed two or more types of ASFs had mean Z-scores 0.26 SD higher $(p<0.01,95 \%$ CI $(0.10,0.42))$ than no ASF consumption (Table 2, Panel A, Nepal, Model 2). In Bangladesh, children who consumed two or more ASFs had mean LAZ scores that were 0.24 SD higher $(p<0.01,95 \%$ CI $(0.14,0.34))$ and a prevalence of stunting $7 \%$ lower $(\mathrm{p}<0.01,95 \% \mathrm{CI}(-0.12,-0.02))$ than those who consumed no ASF (Table 2, Panel A and B, Bangladesh, Model 2). In Uganda, while only $3 \%$ of children consumed two or more types of ASF (Supplementary Table 3), the positive association with LAZ and negative association with stunting was larger in magnitude and statistically significant in children aged 6 to 24 months (Table 2, Panel A and B). The LAZ scores of Ugandan infants, 6 to 12 months, who consumed two or more ASFs was 0.54 SD higher $(\mathrm{p}<0.1,95 \%$ CI $(-0.01,1.09))$ than no ASF consumption (Supplementary Table 6, Panel A, Model 4) and the likelihood of being stunted was lower by $11 \%(\mathrm{p}<0.05,95 \%$ CI $(-0.21,-0.01))$ (Supplementary Table 6, Panel B, Model 4).

\section{Type of ASF and LAZ or Stunting}

In Nepal, meat and dairy consumption were significantly correlated with higher LAZ (meat: $\beta=0.13, \mathrm{p}<0.05,95 \%$ CI $(0.02,0.24)$; dairy: $\beta=0.12, \mathrm{p}<0.05,95 \%$ CI $(0.00,0.23))$ (Table 3). Dairy was of importance among infants (6 to 12 months) and meat among young children (13 to 24 months) (Supplementary Table 7). Dairy products (mainly milk) were the most commonly consumed ASF in Nepal, consumed by roughly $50 \%$ of children compared with $7 \%$ who consumed eggs and 11\% who consumed meat (Supplementary Table 1). In Bangladesh, both 
152 dairy and egg consumption were associated with higher LAZ scores (dairy: $\beta=0.13, p<0.05,95 \%$

153 CI $(0.03,0.24)$; egg: $\beta=0.14, \mathrm{p}<0.1,95 \% \mathrm{CI}(-0.01,0.27))$ and lower stunting (dairy: $\beta=-0.04$,

$154 \mathrm{p}<0.1,95 \%$ CI $(-0.08,0.00)$; egg: $\beta=-0.04, \mathrm{p}<0.1,95 \%$ CI $(-0.09,0.00))$ (Table 3$)$. The results

155 were driven by the 13 to 24 months age group (Supplementary Table 8). In Uganda, consuming

156 dairy $(\beta=0.21, \mathrm{p}<0.05,95 \% \mathrm{CI}(0.02,0.40))$ was associated with higher LAZ scores in children

157 aged 6 to 24 months (Table 3). Fish was also important for higher LAZ scores in children

158 between 13 and 24 months old $(\beta=0.44, \mathrm{p}<0.05,95 \%$ CI $(0.10,0.78))$ (Supplementary Table 9).

\section{Lagged ASF Consumption and LAZ or Stunting}

161 We tested whether anthropometric outcomes were correlated with prior ASF consumption. The

162 lagged analysis was conducted only on the Nepal and Bangladesh data but not Uganda as the 163 surveys were conducted every two years. In Nepal, the LAZ scores of children (6-24 months of 164 age) who consumed any ASF in the prior year were $0.26 \mathrm{SD}$ higher $(\mathrm{p}<0.05,95 \% \mathrm{CI}(0.04,0.47))$ 165 while stunting rates were $10 \%$ lower $(\mathrm{p}<0.05,95 \% \mathrm{CI}(-0.18,-0.02))$ (Table 4, Panels A and B, 166 Nepal Model 1). The association of stunting and number of ASFs consumed in the prior year was 167 strong with stunting being $16 \%$ lower $(\mathrm{p}<0.01,95 \%$ CI $(-0.24,-0.08))$ for those who consumed 168 two or more types of ASF in the prior year relative to those with no ASF consumption (Table 4,

169 Panel B, Nepal Model 2). In Bangladesh, any ASF consumption 6 months prior to LAZ 170 measurement was associated with a 0.14 SD higher LAZ $(p<0.01,95 \%$ CI $(0.04,0.24))$ (Table 4,

171 Panel A, Bangladesh Model 1). LAZ was 0.23 SD higher $(\mathrm{p}<0.01,95 \%$ CI $(0.13,0.33))$ and

172 stunting $10 \%$ lower $(\mathrm{p}<0.01,95 \%$ CI $(-0.14,-0.06))$ in children who had previously consumed

173 two or more ASFs (Table 4, Panels A and B, Bangladesh Model 2). 


\section{Testing lagged and contemporaneous associations}

176 To further assess the relationships, we examined contemporaneous consumption in the same

177 individuals that were included in the lagged consumption models for Nepal and Bangladesh

178 (Supplementary Tables 10 and 11). This was to test for differences in contemporaneous

179 associations in the sub-sample of children included in the lagged models. Finally, we ran

180 regressions that include both the lagged and contemporaneous ASF consumption to determine 181 whether each is related to the outcome in addition to the other (Supplementary Tables 10 and $18211)$.

183

184 In Nepal, in models with both lagged and contemporaneous ASF consumption variables, we 185 found a significant positive association between lagged consumption of any ASF and LAZ $186(\beta=0.26, \mathrm{p}<0.05,95 \% \mathrm{CI}(0.05,0.47))$ but not contemporaneous consumption. Lagged 187 consumption of 1 type of ASF was associated with a significant positive association $(\beta=0.26$, $188 \mathrm{p}<0.05,95 \%$ CI (0.02,0.49)) (Supplementary Table 10, Panel A, Models 5 and 6). Similarly, 189 lagged consumption of any ASF was negatively associated with stunting, while lagged 190 consumption of 1 or 2 or more types of ASF was associated with lowered risk of stunting 191 (Supplementary Table 10, Panel B, Model 5 and 6).

193 In Bangladesh, we found positive statistically significant associations among LAZ, 194 contemporaneous and lagged ASF consumption in the same model (Supplementary Table 11, 195 models 9-12). Past ASF consumption was positively related to LAZ, which was 0.13 SD higher $196(\mathrm{p}<0.05,95 \% \mathrm{CI}(0.03,0.23))$ with any ASF consumption, while contemporaneous ASF 197 consumption was associated with 0.14 higher LAZ $(\mathrm{p}<0.01,95 \%$ CI $(0.06,0.22))$ (Supplementary 
198 Table 11, Panel A, Model 9). The results were not significant using child fixed effects

199 (Supplementary Table 11, Model 10). Consumption of 2 or more ASFs, both lagged and

200 contemporaneous, was significantly associated with 0.19 SD higher LAZ score ( $\mathrm{p}<0.01,95 \%$ CI

$201(0.08,0.31))$ and 0.22 SD higher LAZ-score $(\mathrm{p}<0.01,95 \%$ CI $(0.09,0.35))$, respectively

202 (Supplementary Table 11, Panel A, Model 11). A lower stunting prevalence was found in those

203 consuming two or more ASF types, both six months earlier $(\beta=-0.09, \mathrm{p}<0.01,95 \%$ CI $(-0.13$,-

$2040.05))$ and at the time of measurement $(\beta=-0.07, \mathrm{p}<0.1,95 \%$ CI $(-0.13,0.00))$ (Supplementary

205 Table 11, Panel B, Model 11).

206

207 Discussion

208 Reducing stunting is a key public health policy priority with global targets set for 2025 and

209 beyond ${ }^{24}$. Improving the quality of diets available to children is an important contributor to the

210 achievement of such a target. Given the ongoing global debate about the negative contribution of

211 high meat diets and associated livestock production to greenhouse gas emissions ${ }^{25,26}$, a rigorous

212 evidence-based understanding of the role of meat and other forms of ASF in the diets of

213 undernourished children in resource-poor settings is more critical than ever. The policy goal of

214 optimizing dietary intakes globally to improve human health and the world's chances of

215 mitigating climate change requires a balanced approach; one that seeks to moderate ASF

216 consumption in high- and middle-income settings where levels are already high and growing, as

217 well as increasing intake where diets are still widely deficient in both the quantity and quality of

218 key nutrients.

219 
220 The value of animal sourced foods (ASFs), such as meat, fish, dairy and eggs, in delivering these

221 crucial nutrients along with other bio-active factors like lactoferrin, lysozyme, and growth factors

222 such as insulin-like growth factor-1 has been documented $27,28,29,30$. While sufficient quantity

223 and diversity of non-ASF (i.e. plant-based foods) also contributes many of the micro and

224 macronutrients needed for optimal growth (as shown in our results), ASFs deliver a greater

225 density of many such nutrients (per 1000 calories of food consumed), along with growth factors

226 and certain enzymes that are not available in plants.

227

228 Using longitudinal data, we identified strong correlations between ASF consumption and LAZ or 229 stunting in children aged 6 to 24 months. Both contemporaneous and lagged associations were 230 observed across all three countries. Any ASF consumption was associated with a 0.12 to 0.14 SD 231 higher LAZ across the three countries. The association was stronger with the number of ASFs 232 consumed; children consuming 2 or more types of ASFs demonstrated 0.26 to 0.47 SD higher 233 LAZ scores across all three countries. With respect to the contemporaneous relationship of ASF 234 consumption and stunting, we observed a 7 to $13 \%$ reduction in stunting with the consumption of 2352 or more types of ASF in Bangladesh and Uganda, respectively.

237 The magnitude of lagged ASF consumption effects on LAZ were large, ranging from 0.26 SD in 238 Nepal to 0.23 SD in Bangladesh for 2 or more types of ASF. We found strong correlations where 239 more than one type of ASF was consumed and where lagged effects were taken into account. 240 Dairy had the strongest associations with growth across all three countries with a range of 0.12 2410.22 SD Z-scores, but intake of meat, fish and eggs also played a role. 
243 Some strengths of our study were the use of data from three longitudinal studies that were

244 implemented with rigor, collected exposure data over multiple time points and had large sample

245 sizes, allowing for the assessment of contemporaneous and lagged effects. However, there are

246 some limitations in terms of being unable to control fully for all potential confounders, and the

247 use of different survey time periods across the three country settings. Broad generalizability of

248 our findings is also important to consider: the Nepal study was designed to be nationally

249 representative, while the Bangladesh and Uganda studies were representative of a specific area of

250 the country that was the focus of Feed the Future initiative. Other potential limitations included

251 the lack of information on quantity and caloric value of foods consumed and possible

252 misclassification of "usual diet" caused by the short recall period of 24 hours $31,32$.

254 To our knowledge, this is one of the first studies to rigorously assess empirically the relationship 255 between child growth and both contemporary and prior ASF intake measured over varying time 256 periods. Shapiro et al. (2019) reported that most of the longitudinal cohort studies included in 257 their systematic review found "nonsignificant relations between ASF consumption and indicators 258 of height" ${ }^{33}$. Our analysis focused on diet quality and growth in multiple countries demonstrates 259 significant correlations. This could be a reflection of the management of confounders and use of 260 robust analytical techniques rooted in rigorous econometric methodology.

262 ASFs can play a critical role in the diets of children at risk of the serious physical and cognitive 263 impairment. While increasing the supply and affordability of a wide range of plant-based options 264 to improve diet quality should be part of a public health policy agenda across low-income 265 countries (and for low-income populations in emerging economies and wealthy nations), the 
266 value of raising low intakes of ASFs should not be ignored. Improving efficiencies in livestock,

267 poultry and fish systems is an important agenda, but one that must be pursued alongside policy

268 and programming initiatives to support dietary choices for all households regardless of location

269 and income-level. In rural areas of Uganda, Nepal and Bangladesh, the choice includes optimal,

270 rather than less, intake of diverse forms of ASFs in the diets of young children after they have

271 been exclusively breastfed for 6 months from birth.

272

273 Future research is needed to define what optimal levels of ASF intake are at various stages of

274 child growth for two reasons: to prevent stunting and support ideal linear growth and to assess

275 interactions between linear growth and cognitive outcomes. Additional research is needed to

276 establish the degree to which various ASFs may substitute for each other at different ages and to

277 identify the ideal interactions among ASFs and non-ASFs in the diet, since consumption of other

278 nutrient-rich foods also matters for optimal growth, health and cognition.

279

280 Methods

281 The analysis uses longitudinal panel data from 3 countries. Data from Nepal were derived from

282 the Policy and Science for Health, Agriculture and Nutrition (PoSHAN) research, an annual

283 nationally representative series of surveys (2013-2016) of 5000 households with preschool aged

284 children (6-59 months) in 7 village development committees (VDCs) sampled across three

285 agroecological zones (plains, mountain and hills) in Nepal ${ }^{32}$. Data from Uganda were used from

286 the three biennial (2012-2016) surveys of 3600 households with preschool aged children (6-59

287 months) in 6 districts (4 from northern Uganda and 2 from south-western) in Uganda ${ }^{34}$. Data

288 from Bangladesh were used from the Bangladesh Aquaculture-Horticulture for Nutrition 
Research (2016-2017) that included three bi-annual (2016-2017) surveys of 3167 households with preschool aged children (6-59 months) in three divisions (Dhaka, Khulna and Barisal) of south-western Bangladesh. The three surveys used here were all designed under the auspices of a large-scale global research agenda run by the Feed the Future Innovation Lab for Nutrition. The aim across these individual country studies was to rigorously elucidate pathways by which investments in agriculture may or may not improve diets and nutrition in low-income settings. While each country had particular sub-questions relevant to context, the main community, household and mother-child dyad surveys were similar in design and content, and the core investigative team was the same across all studies. The number of children aged 6-24 months included in this analysis were 1564 in Nepal, 2413 in Bangladesh and 2370 in Uganda.

The outcome variables of interest were length-for-age z-score (LAZ) and whether a child is stunted or not (a binary variable). LAZ was computed from recumbent length (all children are under 2 years of age) and expressed as the number of standard deviations below or above the median of a reference population, adjusted for child age and sex using the WHO defined protocols ${ }^{35}$. We excluded children with missing LAZ scores or with LAZ scores that have biologically implausible values (LAZ $<-5$ or $\mathrm{LAZ}>5$ ). Stunting was computed using a binary variable: a child was classified as stunted if her $L A Z<-2$ and not stunted if her $L A Z \geq-2$, as per WHO 2006 guidelines ${ }^{36}$.

For Nepal and Uganda, we asked if and how often they ate certain food items in the past 24 hours (no quantities were collected) and categorized food items into eight food groups as defined by the WHO: (1) starchy staples; (2) dark green leafy vegetables; (3) vitamin-A rich fruits and 
312 vegetables; (4) other fruits and vegetables; (5) meat and meat products including meat, fish,

313 poultry; (6) eggs; (7) dairy; and (8) legumes, nuts and seeds. The Bangladesh panel used a

314 classification of foods into six groups: (1) starchy staples; (2) fruits and vegetables; (3) meat and

315 meat products including meat, fish, poultry; (4) eggs; (5) dairy; and (6) legumes, nuts and seeds.

316 We aggregated meat and meat products, eggs and dairy for the variable 'animal sourced foods'

317 (ASFs). In addition, we generated binary variables for each type of ASF consumed (e.g. meat

318 and meat products, dairy, eggs, and fish in the case of Bangladesh only) for exploration in

319 separate models. The survey provided individual- and household-level information that were

320 included in the analysis including age, gender, number of illnesses in the past 7 days, maternal

321 height and education, and household sanitation status (presence or absence of a latrine).

322

323 Supplemental Tables 1-3 report for each survey summary statistics of the variables used in the

324 analysis. We computed summary statistics for the full samples, as well as disaggregated into two 325 age groups, 6 to 12 months and 13 to 24 months.

326

327 Although there was variation in mean LAZ scores across the three country samples, they share a 328 common pattern in that LAZ scores decrease with age, a phenomenon that is common across 329 most low- and middle-income countries ${ }^{37}$. The same pattern holds for stunting prevalence, 330 which is higher in the older age groups across all survey data sets. The average prevalence of 331 stunting ranges from 26\% in Bangladesh and Uganda to 30\% in Nepal.

333 The consumption of ASFs also varies widely across the three countries. Uganda has the lowest 334 average share of children consuming any ASF daily (23\%), and only $3 \%$ of those children 
335 consume two or more types of ASFs. By contrast, Bangladesh has the largest average share of

336 children who consume ASFs - 76\% have consumed any ASFs, and 36\% of them have consumed

337 two or more types of ASFs. Additionally, there are differences across countries in the types of

338 ASF most likely to be consumed: dairy in Nepal and Uganda compared to flesh foods (meat) in

339 Bangladesh. Eggs are the least common form of ASF consumed by children in all three surveys.

341 We estimate the following model to examine the relationships between ASF consumption and 342 child stunting:

$$
=\beta_{1} A S F \text { consumption }_{i, t}
$$

347 Child outcome is either the length-for-age z-score of the child $i$ in survey round $t$, or a binary 348 variable that indicates whether the child is stunted or not. We are interested in the association 349 between the two outcomes and children's consumption of ASFs, which we measure in two ways:

350 (1) a binary variable that indicates whether a child consumed any ASFs or not, and (2) two 351 binary variables that indicate whether a child consumed one type of ASF, or two or more types 352 of ASFs.

354 Models were estimated using fixed effects (FE) panel regression. This implies a linear 355 probability model with fixed effects for the binary outcome 'stunting'. Since we include fixed 356 effects, a binary choice model like Probit would suffer from the incidental parameters problem ${ }^{38}$. 357 We control for confounding factors potentially correlated with ASF consumption while also 
358 possibly affecting outcome variables. To adjust for potential contribution to the diet, and thus to

359 nutritional status from foods other than ASFs, we include consumption of items from all the

360 other food groups in the same 24-hour recall period $j$. We also control for a vector of individual

361 characteristics, $\mathbf{z}_{i, t}^{\prime}$, that includes child characteristics (age, gender, whether the child recently

362 had diarrhea), maternal height and education, and household sanitation (whether the household

363 has an improved latrine). We also tested alternative models that included the household's wealth

364 index instead of mother's education and household sanitation. Most, but not all results were

365 robust to the alternative specification. In our preferred specification we used education and

366 sanitation which are correlated with the household's wealth index but more proximal correlates

367 of stunting. We further include district*survey-round fixed effects, $\Phi_{d, t}$, which control for any

368 local temporal shocks that are common to all children in a single district-round. This is important

369 to capture variation at the local level in the food prices, market availability etc., and also the

370 health environment and other observed or unobserved local conditions that affect children's

371 outcomes. Where the data permit, we ran additional regressions that included child fixed effects

372 to control for characteristics of a child that do not change over time, such as maternal health and

373 nutrition during pregnancy or birth outcome, together with survey round fixed effects to account

374 for temporal shocks that affect all children in a survey.

375

376 A child's diet today can have a lagged effect on her/his subsequent growth, which we test by

377 regressing a child's outcome of interest on her consumption of ASFs and foods from other

378 groups in the previous period: 


\section{Child outcome $_{i, t}$}

$$
=\beta_{1} A S F \text { consumption }_{i, t-1}
$$

$+\sum_{j} \gamma_{j}$ Consumption of foods from group $j_{i, t-1}+\mathbf{z}_{i, t}^{\prime} \delta+\lambda \Phi_{d, t}+\varepsilon_{i, t}$

Depending on the dataset used, the lags range from six months to one year. Statistical analysis was performed using Stata 15.1 (StataCorp LP).

\section{Acknowledgements:}

The authors express gratitude to USAID's Bureau of Resilience and Food Security, the US Missions in all 3 countries included in this study, as well as USAID staff Dr. Maura Mack, Dr. Ahmed Kablan, Mr. Debendra Adhikari, and Dr. Osagie Aimiuwu. We also thank the study participants without whom this research would not have been possible. Support for the research was provided by the Feed the Future Innovation Lab for Nutrition, which is funded by the United

States Agency for International Development (USAID), award AID-OAA-L-10-00006 (Nepal and Uganda), award AID-OAA-LA-14-00012 (Bangladesh) to the Friedman School of Nutrition Science and Policy at Tufts University. The opinions expressed herein are solely those of the authors.

\section{Author Contributions:}

PW conceived the study. SZ, PW and SG developed the methodology and SZ conducted the analysis; PW, SG and RS helped to refine the methodology and analytical method; SZ, PW and SG interpreted the results; SZ, PW and SG developed early drafts of the paper, and RS contributed to writing, reviewing and editing early drafts; SM, ATL, BB, SG designed the 
401 surveys and supervised the data collection; GS, SM, BB and RS led data collection; SM, KMH, 402 LL, and GN performed the data curation. All authors reviewed the final manuscript.

403

404 Competing Interests Statements: The authors declare no competing interests.

405

406 References

4071 UNICEF, WHO, IBRD \& Bank, W. Levels and trends in child malnutrition: key findings

408 of the 2019 Edition of the Joint Child Malnutrition Estimates. . 16 (UNICEF, WHO, IBRD,

$409 \quad$ World Bank, Geneva, 2019).

4102 Kinyoki, D. K. et al. Mapping child growth failure across low- and middle-income 411 countries. Nature 577, 231-234, doi:10.1038/s41586-019-1878-8 (2020).

4123 Aguayo, V. M. \& Menon, P. Stop stunting: improving child feeding, women's nutrition and 413 household sanitation in South Asia. Matern Child Nutr 12 Suppl 1, 3-11, 414 doi:10.1111/mcn.12283 (2016).

4154 Black, R. E. et al. Maternal and child undernutrition and overweight in low-income and 416 middle-income countries. The Lancet 382, 427-451 (2013).

4175 Ahmed, T. et al. in Hunter's Tropical Medicine and Emerging Infectious Diseases (eds 418 Ryan ET. et al.) 1034-1041 (2020).

4196 Jeffery, K., Chatterjee, I., Lavin, T. \& Li, I. W. Young lives and wealthy minds: The nexus 420 between household consumption capacity and childhood cognitive ability. Economic 421 Analysis and Policy 65, 89-104, doi:https://doi.org/10.1016/j.eap.2019.11.002 (2020). 
4227 Han, V., Radford, B. \& Awamleh, Z. in Maternal-Fetal and Neonatal Endocrinology 423 Physiology, Pathophysiology, and Clinical Management (eds Kovacs CS \& Deal CL) $424 \quad(2020)$.

4258 Bhutta, Z. A. et al. Severe childhood malnutrition. Nature Reviews Disease Primers 3, $426 \quad$ 17067, doi:10.1038/nrdp.2017.67 (2017).

4279 Ghosh, S., Suri, D. \& Uauy, R. Assessment of protein adequacy in developing countries: 428 quality matters. British Journal of Nutrition 108, S77-S87 (2012).

$42910 \quad$ Krasevec, J., An, X., Kumapley, R., Begin, F. \& Frongillo, E. A. Diet quality and risk of 430 stunting among infants and young children in low- and middle-income countries. Matern Child Nutr 13 Suppl 2, doi:10.1111/mcn.12430 (2017).

43211 Headey, D., Hirvonen, K. \& Hoddinott, J. Animal Sourced Foods and Child Stunting. 433 American Journal of Agricultural Economics 100, 1302-1319, doi:10.1093/ajae/aay053 $434 \quad$ (2018).

43512 Hoppe, C., Molgaard, C. \& Michaelsen, K. F. Cow's milk and linear growth in 436 industrialized and developing countries. Annu Rev Nutr 26, 131-173, 437 doi:10.1146/annurev.nutr.26.010506.103757 (2006).

43813 Ghosh, S., Suri, D. \& Griffiths, J. Dairy consumption is associated with a lower risk of 439 stunting in Ethiopian children 6-24 months of age (620.10). The FASEB Journal 28, $440 \quad 620.610(2014)$.

44114 Dror, D. K. \& Allen, L. H. Dairy product intake in children and adolescents in developed 442 countries: trends, nutritional contribution, and a review of association with health outcomes. Nutr Rev 72, 68-81, doi:10.1111/nure.12078 (2014). 
44415 Mahmudiono, T., Sumarmi, S. \& Rosenkranz, R. R. Household dietary diversity and child 445 stunting in East Java, Indonesia. Asia Pac J Clin Nutr 26, 317-325, doi:10.6133/apjen.012016.01 (2017).

44716 Neumann, C. G. et al. Animal Source Foods Improve Dietary Quality, Micronutrient Design and Baseline Findings. The Journal of Nutrition 133, 3941S-3949S, doi:10.1093/jn/133.11.3941S (2003).

45117 Krebs, N. F. et al. Meat consumption is associated with less stunting among toddlers in 452 four diverse low-income settings. Food Nutr Bull 32, 185-191, 453 45418 Marinda, P. A., Genschick, S., Khayeka-Wandabwa, C., Kiwanuka-Lubinda, R. \& 455 456 457 45819 Kaimila, Y. et al. Consumption of Animal-Source Protein is Associated with Improved 459 460

46120 Iannotti, L. L. et al. Eggs in Early Complementary Feeding and Child Growth: A

462 Randomized Controlled Trial. Pediatrics 140, e20163459, doi:10.1542/peds.2016-3459 463 Height-for-Age z Scores in Rural Malawian Children Aged 12-36 Months. Nutrients 11, 480 (2019).

Thilsted, S. H. Dietary diversity determinants and contribution of fish to maternal and under-five nutritional status in Zambia. PLOS ONE 13, e0204009, doi:10.1371/journal.pone.0204009 (2018). (2017).

46421 Shivakumar, N. et al. Protein-quality evaluation of complementary foods in Indian 465 children. The American Journal of Clinical Nutrition 109, 1319-1327, 466 doi:10.1093/ajen/nqy265 (2019). 
46722 Iannotti, L. L. et al. Egg intervention effect on linear growth no longer present after two years. Maternal \& Child Nutrition 16, e12925, doi:10.1111/mcn.12925 (2020).

46923 Stewart, C. P. et al. The effect of eggs on early child growth in rural Malawi: the Mazira 470 Project randomized controlled trial. The American Journal of Clinical Nutrition 110, 1026-

471 1033, doi:10.1093/ajen/nqz163 (2019).

47224 de Onis, M. \& Branca, F. Childhood stunting: a global perspective. Maternal \& Child 473 Nutrition 12, 12-26, doi:10.1111/mcn.12231 (2016).

47425 Willett, W. et al. Food in the Anthropocene: the EAT\&\#x2013;<em $>$ Lancet $</$ em $>$ 475 Commission on healthy diets from sustainable food systems. The Lancet 393, 447-492, 476 doi:10.1016/S0140-6736(18)31788-4 (2019).

47726 Swinburn, B. A. et al. The Global Syndemic of Obesity, Undernutrition, and Climate 478 Change: $<\mathrm{em}>$ The Lancet $</ \mathrm{em}>$ Commission report. The Lancet 393, 791-846, 479 480 doi:10.1016/S0140-6736(18)32822-8 (2019). Bulletin 34, 268-271, doi:10.1177/156482651303400224 (2013).

48428 Milward, D. Nutrition, infection and stunting: The roles of deficiencies of individual 485 nutrients and foods, and of inflammation, as determinants of reduced linear growth of 486 children. Nutrition Research Reviews 30, 50-72 (2017).

48729 Murphy, S. P. \& Allen, L. H. Nutritional Importance of Animal Source Foods. The Journal 488 of Nutrition 133, 3932S-3935S, doi:10.1093/jn/133.11.3932S (2003). 
48930 Hoppe, C. et al. Animal protein intake, serum insulin-like growth factor I, and growth in 490 healthy 2.5-y-old Danish children. The American Journal of Clinical Nutrition 80, 447452, doi:10.1093/ajcn/80.2.447 (2004).

49231 Thorne-Lyman, A., Spiegelman, D. \& Fawzi, W. W. Is the strength of association between indicators of dietary quality and the nutritional status of children being underestimated? Maternal \& Child Nutrition 10, 159 (2014).

49532 Klemm, R. et al. Pathways from agriculture-to-nutrition: design and conduct of the national $496 \quad$ PoSHAN surveys of Nepal. (2018).

49733 Shapiro, M. J. et al. A Systematic Review Investigating the Relation Between Animal498 499 500

$50134 \quad$ NIL. Innovation Lab for Nutrition Annual Report. 81 ( 2018).

50235 WHO. WHO Anthro (version 3.1, June 2010) and macros,

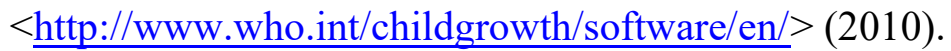

WHO. WHO Child Growth Standards: Methods and development: Length/height-for-age, 505 weight-for-age, weight-for-length, weight-for-height and body mass index-for-age., $<\underline{\text { http://www.who.int/childgrowth/publications/technical report pub/en/index.html }>}$ (2007).

50837 Leroy, J. L., Ruel, M., Habicht, J.-P. \& Frongillo, E. A. Using height-for-age differences 509 (HAD) instead of height-for-age z-scores (HAZ) for the meaningful measurement of 
51238 Greene, W. Estimating econometric models with fixed effects. Department of Economics, $513 \quad$ Stern School of Business, New York University (2001). 


\section{Tables}

Table 1: Descriptive statistics by country

\begin{tabular}{|c|c|c|c|c|c|c|}
\hline & \multicolumn{2}{|c|}{ Nepal } & \multicolumn{2}{|c|}{ Bangladesh } & \multicolumn{2}{|c|}{ Uganda } \\
\hline & Mean & Std. Dev. & Mean & Std. Dev. & Mean & Std. Dev. \\
\hline $\begin{array}{l}\text { Length for Age Z-score (6-24 } \\
\text { months) }\end{array}$ & -1.41 & 1.19 & -1.28 & 1.14 & -1.15 & 1.45 \\
\hline $\begin{array}{l}\text { Mean percent children stunted } \\
\text { (6-24 months) }\end{array}$ & $30 \%$ & $46 \%$ & $26 \%$ & $44 \%$ & $26 \%$ & $44 \%$ \\
\hline $\begin{array}{l}\text { Mean percent children (6-24 } \\
\text { months) consuming any ASF } \\
\text { in past } 24 \text { hours }\end{array}$ & $57 \%$ & $50 \%$ & $76 \%$ & $43 \%$ & $23 \%$ & $42 \%$ \\
\hline Number of observations & & 1564 & & 13 & & 370 \\
\hline
\end{tabular}

The table reports summary statistics of the datasets used in the main regressions (Tables 2 and 3 ). For each sample

(Nepal, Bangladesh, and Uganda), the unit of the data is a child-survey wave pair. The datasets are unbalanced panels consisting of three annual surveys in Nepal (2014-2016), three bi-annual surveys in Bangladesh (2016-2017), and three biennial surveys in Uganda (2012-2016). The outcome variable Length for Age Z-score was computed from recumbent length (all children are under 2 years of age) and expressed as the number of standard deviations below or above the median of a reference population, adjusted for child age and sex using the WHO defined protocols ${ }^{35}$. We excluded children with missing LAZ scores or with LAZ scores that have biologically implausible values (LAZ<-5 or LAZ $>5$ ). Stunting was computed using a binary variable: a child was classified as stunted if her LAZ $<-2$ and not stunted if her LAZ $\geq-2$, as per WHO 2006 guidelines ${ }^{36}$. We use a binary variable that indicates whether a child consumed any ASFs or not, where we aggregated meat and meat products, eggs and dairy for the variable 'animal sourced foods' (ASFs). More detailed summary statistics are reported in the Supplementary Tables. 
Table 2: Association between LAZ or stunting in children (6-24 months of age) and contemporaneous ASF consumption

\begin{tabular}{|c|c|c|c|c|c|c|}
\hline & \multicolumn{2}{|c|}{ Nepal } & \multicolumn{2}{|c|}{ Bangladesh } & \multicolumn{2}{|c|}{ Uganda } \\
\hline & (1) & (2) & (1) & (2) & (1) & (2) \\
\hline \multicolumn{7}{|c|}{ PANEL A: LAZ AND CONTEMPORANEOUS ASF CONSUMPTION: AGES 6-24 MONTHS } \\
\hline \multirow[t]{2}{*}{ Child consumed any ASF } & $0.120 *$ & & $0.116^{* * *}$ & & $0.138 *$ & \\
\hline & $(0.063)$ & & $(0.033)$ & & $(0.072)$ & \\
\hline \multirow[t]{2}{*}{ Child consumed 1 type of ASF } & & 0.092 & & 0.039 & & 0.099 \\
\hline & & $(0.066)$ & & $(0.041)$ & & $(0.076)$ \\
\hline \multirow[t]{2}{*}{ Child consumed 2 or more types of ASF } & & $0.258 * * *$ & & $0.241 * * *$ & & $0.467 * * *$ \\
\hline & & $(0.070)$ & & $(0.048)$ & & $(0.145)$ \\
\hline $\mathrm{N}$ & 1564 & 1564 & 2413 & 2413 & 2370 & 2370 \\
\hline adjusted R-square & 0.288 & 0.289 & 0.169 & 0.174 & 0.232 & 0.234 \\
\hline \multicolumn{7}{|c|}{ PANEL B: STUNTING AND CONTEMPORANEOUS ASF CONSUMPTION: AGES 6-24 MONTHS } \\
\hline \multirow[t]{2}{*}{ Child consumed any ASF } & -0.021 & & -0.027 & & $-0.030^{*}$ & \\
\hline & $(0.020)$ & & $(0.020)$ & & $(0.018)$ & \\
\hline \multirow[t]{2}{*}{ Child consumed 1 type of ASF } & & -0.014 & & 0.000 & & -0.018 \\
\hline & & $(0.018)$ & & $(0.021)$ & & $(0.020)$ \\
\hline \multirow[t]{2}{*}{ Child consumed 2 or more types of ASF } & & -0.059 & & $-0.070 * * *$ & & $-0.134 * * *$ \\
\hline & & $(0.040)$ & & $(0.023)$ & & $(0.039)$ \\
\hline $\mathrm{N}$ & 1564 & 1564 & 2413 & 2413 & 2370 & 2370 \\
\hline adjusted R-square & 0.176 & 0.176 & 0.110 & 0.115 & 0.157 & 0.159 \\
\hline \multicolumn{7}{|c|}{ The table reports estimation results using FE panel regressions. The outcome variable in Panel A is child's LAZ and a dummy variable equal to 1 if the child is stunted in Panel B. } \\
\hline \multicolumn{7}{|c|}{$\begin{array}{l}\text { Co-variates and control variables: child consumed starchy staples, dark green leafy vegetables, vitamin A rich fruits and vegetables, other fruits and vegetables, legumes, nuts and } \\
\text { seeds, child's age, age squared, age cube, a dummy variable equal to } 1 \text { if the child is a girl, a dummy variable equal } 1 \text { if the child had diarrhea in the past two weeks, the caregiver's }\end{array}$} \\
\hline seeds, child's age, age squared, age cube, a du & ual to 1 if th & sirl, a dumm & le equal 1 if $t$ & ad diarrhea in & weeks, th & er's \\
\hline \multicolumn{7}{|c|}{ years of education, the mothers height, a dummy equal to 1 if the household has an improved latrine. Data sources are POSHAN (Nepal), BAHNR (Bangladesh), Uganda Panel } \\
\hline \multicolumn{7}{|c|}{ (Uganda). All regressions include control variables and District X wave fixed effects. Standard errors clustered by district are reported in parentheses. } \\
\hline${ }^{*} \mathrm{p}<0.1, * * \mathrm{p}<0.05, * * * \mathrm{p}<0.01$ & & & & & & \\
\hline
\end{tabular}


Table 3: Association between LAZ or stunting in children (6-24 months of age) and contemporaneous ASF consumption by type: Nepal, Bangladesh and Uganda

\begin{tabular}{llll}
\hline & \multicolumn{2}{c}{ Nepal } & \multicolumn{2}{c}{ Bangladesh } & \multicolumn{2}{c}{ Uganda } \\
\hline PANEL A: LAZ AND CONTEMPORANEOUS ASF CONSUMPTION BY TYPE \\
\hline Child consumed meat/meat products & $0.128^{* *}$ & 0.089 & 0.110 \\
Child consumed eggs & $(0.047)$ & $(0.076)$ & $(0.108)$ \\
& 0.018 & $0.135^{*}$ & $-0.497^{* * *}$ \\
Child consumed dairy & $(0.093)$ & $(0.067)$ & $(0.186)$ \\
& $0.118^{* *}$ & $0.133^{* *}$ & $0.211^{* *}$ \\
Child consumed fish & $(0.050)$ & $(0.049)$ & $(0.098)$ \\
& 0.019 & 0.037 & 0.166 \\
N & $(0.117)$ & $(0.040)$ & $(0.135)$ \\
adjusted R-square & 1564 & 2413 & 2237 \\
\hline
\end{tabular}

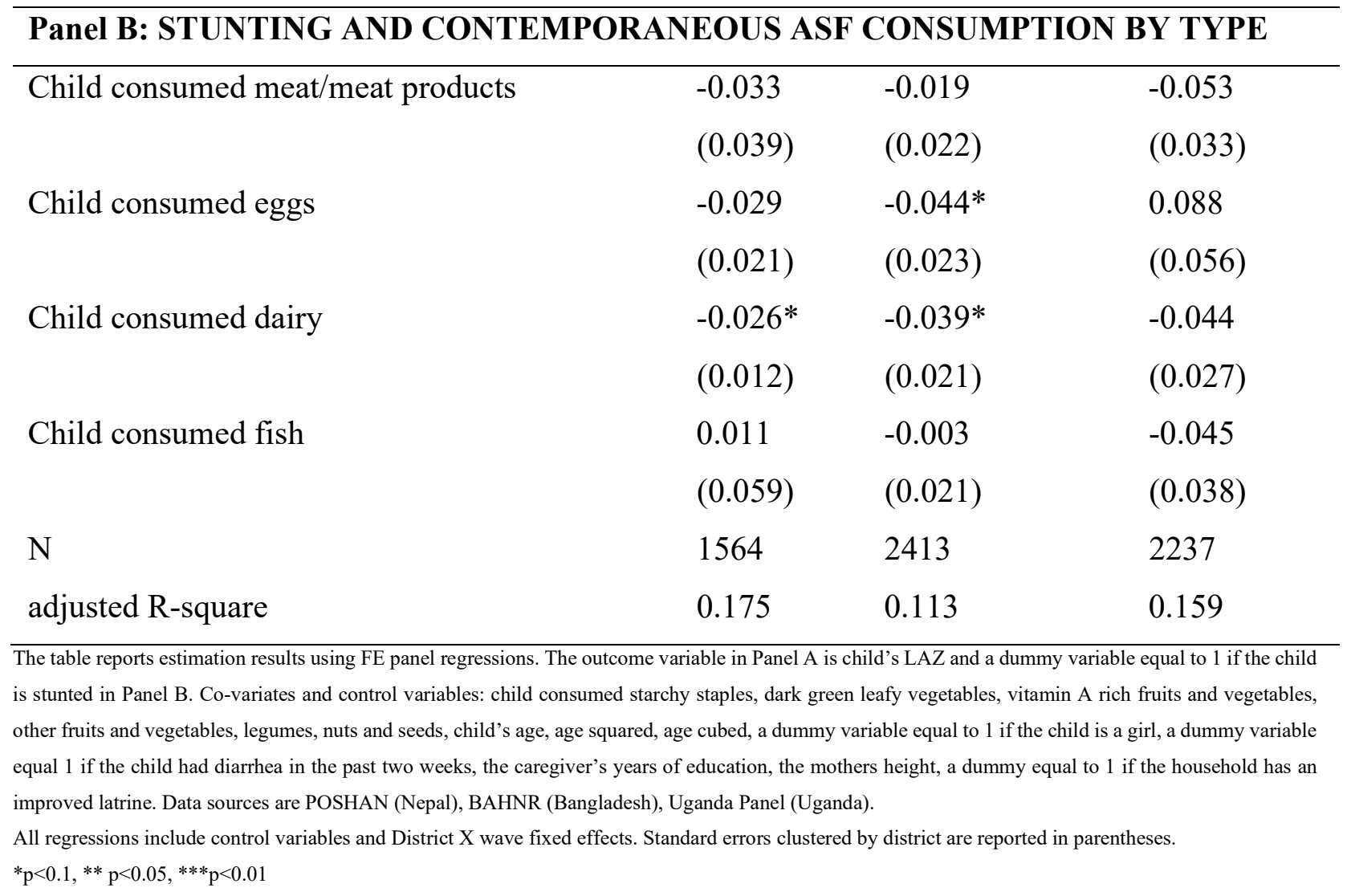


Table 4: Association of LAZ or stunting in children (aged 6-24 months) and lagged ASF consumption in Nepal and Bangladesh

Nepal

(1)
(2)
Bangladesh

(1)

(2)
PANEL A: LAZ AND LA
Child consumed any ASF
$0.257 * *$
$0.140 * * *$
(0.093)
(0.049)
Child consumed 1 type of ASF
$0.254 * *$
0.095
(0.102)
(0.061)
Child consumed 2 or more types of ASF
0.283
$0.231 * * *$
(0.162)
$\mathrm{N}$
787
787
1381
1381
adjusted R-square
0.207
0.206
0.170
0.172

\section{PANEL B: STUNTED AND LAGGED ASF CONSUMPTION: AGES 6-24 MONTHS}

\begin{tabular}{lllll}
\hline Child consumed any ASF & $-0.099 * *$ & \multicolumn{2}{c}{-0.033} \\
& $(0.034)$ & \multicolumn{2}{c}{$(0.024)$} & -0.001 \\
Child consumed 1 type of ASF & & $-0.092^{* *}$ & $(0.029)$ \\
& & $(0.039)$ & & $-0.099 * * *$ \\
Child consumed 2 or more types of ASF & & $-0.159 * * *$ & $(0.019)$ \\
N & & $(0.036)$ & & 1381 \\
adjusted R-square & 787 & 787 & 1381 & 0.119
\end{tabular}

The table reports estimation results using FE panel regressions. The outcome variable in Panel A is child's LAZ and a dummy variable equal to 1 if the child is stunted in Panel B. Co-variates and control variables: child consumed starchy staples, dark green leafy vegetables, vitamin A rich fruits and vegetables, other fruits and vegetables, legumes, nuts and seeds, child's age, age squared, age cubed, a dummy variable equal to 1 if the child is a girl, a dummy variable equal 1 if the child had diarrhea in the past two weeks, the caregiver's years of education, the mothers height, a dummy equal to 1 if the household has an improved latrine. Data sources are POSHAN (Nepal), BAHNR (Bangladesh), Uganda Panel (Uganda). All regressions include control variables and District $\mathrm{X}$ wave fixed effects. Standard errors clustered by district are reported in parentheses. $* \mathrm{p}<0.1, * * \mathrm{p}<0.05, * * * \mathrm{p}<0.01$ 


\section{Supplementary Files}

This is a list of supplementary files associated with this preprint. Click to download.

- SupplementarytablesZahariaetal.pdf 